\title{
LA SUPERVISIÓN EDUCATIVA Y SU RELACIÓN CON EL DESEMPEÑO DOCENTE EN LAS INSTITUCIONES EDUCATIVAS DE LA RED N08 PUENTE PIEDRA -
}

LIMA- 2014

EDUCATIONAL SUPERVISION AND ITS RELATIONSHIP WITH TEACHING PERFORMANCE IN EDUCATIONAL INSTITUTIONS OF THE NETWORK $N{ }^{\circ} 08$

PUENTE PIEDRA - LIMA- 2014

A SUPERVISÃO EDUCACIONAL E SUA RELAÇÃO COM O DESEMPENHO DOCENTE NAS INSTITUIÇÕES DE EDUCAÇÃO DA REDE N ${ }^{\circ} 08$ PUENTE PIEDRA

ISSN: 2617-619X

Resumen: La investigación científica que hemos realizado es básica, correlacional causal no experimental, los factores de estudio son dos: gestión educativa y desempeño docente. La población y muestra estuvo conformada por docente y jerárquico de la RED 08 de Puente Piedra. El diseño de la investigación es transeccional, correlacional, causal, bivariada, transversal.

Para la recolección de datos se confeccionaron dos cuestionarios estructurados, los instrumentos cumplen con las cualidades de validez y confiabilidad. El estudio

\footnotetext{
${ }^{1}$ Universidad Nacional Mayor de San Marcos

2 Universidad Nacional Mayor de San Marcos
} 
plantea la siguiente hipótesis de investigación: Existe relación significativa entre la gestión educativa y el desempeño docente en las instituciones educativas de la Red Nº8 - Puente Piedra -Lima - 2014. Los resultados obtenidos nos permiten rechazar la hipótesis nula y aceptar la hipótesis investigadas, al aplicar la formula Rho de Spearman con un nivel de significancia del 95\% encontramos que el coeficiente de correlación Rho de Spearman es de 0,903 y el p_valor es de 0,000 por lo tanto establecemos que tiene un nivel correlación muy bueno, vemos que existe una relación directa y un nivel de significancia menor que 0,05 por tal motivo se rechaza la hipótesis Nula y se acepta la alterna.

Palabras Clave: La gestión educativa-Desempeño docente -Instituciones Educativas de la RED 08 de Puente Piedra - Lima

\begin{abstract}
The research we have done is basic causal correlational experimental study factors are two educational supervision and teacher performance. The population sample consisted of 34 teachers and hierarchical RED Pedra Bridge 08. The research design is transactional, correlational, causal, bivariate cross.
\end{abstract}

For data collection two structured questionnaires were prepared, the instruments meet the qualities of validity and reliability. The study raises the following research hypothesis: There is significant relationship between educational supervision and teacher performance in the educational institutions of the Red $\mathrm{N}$ ○ 08 - Stone Bridge-Lima - 2014. The results allow us to reject the null hypothesis and accept the hypothesis investigated by applying the formula of Spearman Rho with a significance level of $95 \%$ found that the correlation coefficient Spearman Rho is 0.903 and the value is 0.000 p_ by thus we establish that correlation has a very good level, we see that there is a direct and a significance level of less than 0.05 for that reason we reject the null hypothesis and accept the alternative.

Keywords: Performance Monitoring teacher-education-Educational Institutions RED Stone Bridge 08 - Lima 
Introducción:_El término gestión educativa tiene significados diferentes, toda persona que lee o escucha esta palabra, la interpreta según su experiencia, necesidades y propósitos. la gestión educativa puede considerarla como una fuerza positiva para mejorar un programa o proceso dentro de una empresa. en el ámbito educativo un maestro puede verla como una amenaza a su individualidad; otro puede buscarla como una fuente de ayuda y de apoyo.

La necesidad de gestión educativa surge cuando se reconoce la diferencia entre el modo como son las cosas y el modo como deben de ser, uno de los métodos más eficaces para analizar y mejorar las situaciones, es tener una visión clara y objetiva de lo que está sucediendo y es esto lo que se busca en la educación, ya que el proceso educativo no puede quedar librado a su suerte y a la expectativa de si resulta o no.

La buena enseñanza no puede depender de maestros excepcionales o de la fortuna, es entonces cuando la gestión educativa se impone como órgano interesado en el desempeño de la escuela, para que la acción de ésta mejore constantemente y los buenos resultados estén garantizados de manera objetiva y científica.

En términos generales, la supervisión es ayuda para mejorar. ocurre cuando se ayuda a los maestros a dar un paso adelante, siendo la tarea de la gestión educativa el mejorar la situación de aprendizaje, realizando una actividad de servicio que apoya a los maestros para que realicen mejor su función.

El presente trabajo trata de la gestión educativa y el buen desempeño, intenta definir lo que es y cómo ha evolucionado este concepto, se mencionan las funciones y etapas de la supervisión y su relación con el mejoramiento de la calidad del producto de la educación. la importancia que reviste este tema actualmente es grande, y hoy más que nunca debe conocerse sobre lo que es la gestión y sus beneficios, porque a diario encontramos una exigencia por la productividad y calidad en el ámbito educativo y en los docentes recae mucha 
de la responsabilidad del logro de esa calidad porque la misma se inicia en la escuela con el proceso enseñanza-aprendizaje que debe ser mejorado constantemente y una actividad que nos apoya y asiste para el logro de este fin es precisamente la gestión educativa

Las escasas investigaciones que se realizan en los niveles Gestión educativa a nivel nacional, están referidas sobre realidades superficiales a las instituciones educativas, pocas veces se realizan unas autoevaluaciones especializadas acerca de los niveles de calidad que sirva para retroalimentar el proceso de desempeño docente en la perspectiva del mejoramiento continuo para la formación de directores a nivel de Ministerio de Educación.

Además es importante el estudio ya que permitiría realizar algunas recomendaciones que tiendan a controlar los factores negativos que limitan elevar la calidad de formación de los docentes. Especialmente buscaremos sugerencias para mejorar el contexto administrativo como factores favorables que promuevan la calidad educativa.

Rincón (2005) en su tesis: Relación entre estilo de liderazgo del director y desempeño de docentes del Valle del Chumbao de la Provincia de Andahuaylas" concluye que 1.- Los datos nos evidencian que entre el estilo de liderazgo del director y el desempeño docente existe un alto grado de correlación (0,76 de correlación de acuerdo al coeficiente de personas es una correlación positiva considerable) en las instituciones educativas del Valle del Chumbao de la provincia de Andahuaylas. Esto se puede explicar por la relevancia que tiene la dirección de una institución educativa el cual depende deducir que el desempeño docente depende del estilo de liderazgo del director lo cual demuestra la hipótesis propuesta.

Se ha hallado que en las Instituciones educativas del Valle del Chumbao los estilos de director que predomina son el estilo anárquico y el autoritario, lo cual evidencia que no ha habido innovaciones significativas en el estilo de dirección 
pues existe evidencia de que se continúa con estilos tradicionales de gestión por parte de los directores de las instituciones educativas. Los datos relacionados al desempeño de los docentes nos permiten concluir que en la mayoría de las instituciones educativas del Valle del Chumbao existe un bajo nivel de desempeño docente por cuanto está afectado por el estilo de liderazgo de los directores.

El estudio permite concluir que el estilo de liderazgo adecuado del director que puede incrementar el desempeño de los docentes es el estilo democrático y situacional del director que motiva a los docentes, y prioriza

Fernández (1992) en un estudio titulado "Relación entre el Estilo Gerencial del Personal Directivo de las Escuelas Básicas de Altagracia de Orituco correspondientes a la I y II Etapa, y el Clima Organizacional de dichas instituciones", enfatiza la necesidad de relacionar el estilo gerencial del personal directivo de las Escuelas Básicas de Altagracia de Orituco con el clima organizacional de las mencionadas instituciones. Para el logro de este objetivo, se realizó un análisis profundo de tres tipos de estilos gerenciales: autoritario, democrático y Laissez - Faire, de los tipos de comunicación y de las teorías de motivación que se consideraron más adaptadas al trabajo en cuestión. La investigación se enmarcó dentro de la modalidad de trabajo de campo, según la dimensión cronológica es descriptiva. En atención al método se utilizó el método correlacional, específicamente la prueba de asociación Chi cuadrado. Para la recolección de los datos se utilizó un cuestionario estructurado con 50 ítems que se aplicó por separado a dos estratos muestrales: El primero conformado por los 17 directivos de las escuelas básicas, el segundo formado por el $50 \%$ de los docentes de aula los cuales fueron seleccionados al azar. Los resultados de la investigación determinaron: Que el estilo gerencial predominante en las instituciones de Educación Básica de Altagracia de Orituco, es el Laissez - Faire y que la comunicación informal predominó sobre los demás tipos de comunicación. Se indica además que no hubo una definición clara sobre las clases de comunicación: ascendente, descendente y horizontal. Los 
procedimientos estadísticos correlacionales, revelan que existe relación entre el estilo Laissez - Faire encontrado como estilo gerencial predominante y la comunicación informal. Porcentualmente se encontró que existe relación entre el estilo gerencial y la subdimensión motivación, llegándose a la conclusión de que los directivos de estos centros no motivan al personal a participar.

Ascanio (1995), en su estudio titulado "El Liderazgo del Supervisor y la Motivación hacia el Mejoramiento Profesional en los Docentes que laboran en la I y II Etapa de Educación Básica en Altagracia de Orituco, Estado Guárico”, plantea la necesidad de analizar el rol de líderes cumplido por los supervisores y su relación con la motivación hacia el mejoramiento profesional. La investigación se llevó a cabo en las instituciones donde se imparte Educación Básica de la I y II Etapa en Altagracia de Orituco, Estado Guárico. Para el estudio en referencia se utilizó un diseño de investigación de campo, ex post - facto, no experimental, transaccional, descriptivo. Se elaboró para la recopilación de los datos referidos, un cuestionario integrado por 27 ítems con cuatro alternativas cada uno; el mismo fue aplicado a una muestra representativa del 36 \%. Los resultados evidencian que los supervisores cumplen en forma muy deficiente, su rol de líderes motivadores de los docentes, para que éstos se dediquen a trabajar por su mejoramiento profesional. De igual forma, los resultados manifiestan que los docentes poseen un alto grado de motivación hacia el desarrollo de actividades de mejoramiento profesional, lo cual puede ser usado como base para que los Supervisores diseñen y desarrollen estrategias que permitan lograr un personal con un alto nivel de capacitación, y que al mismo tiempo, permita a dichos supervisores, cumplir su rol de líderes a cabalidad.

Luis Arturo Lemus: "Supervisión quiere decir coordinar, estimular y dirigir el desenvolvimiento de los profesores, para que por medio de ellos, se estimule a cada individuo a través del ejercicio de su talento hacia la más completa y más inteligente participación en la sociedad a la cual pertenece". 
Imódeo Nérici (1975) "La supervisión escolar puede sintetizarse como asistencia a las actividades docentes, de manera de darles coordinación, unidad y continuidad, para que la escuela alcance con mayor eficiencia sus objetivos". “...la supervisión deberá entenderse como los esfuerzos llevados a cabo por la escuela con el objeto de llevar a los maestros y demás personas que tienen a su cargo el desarrollo y la conducción del proceso educativo a ejercer un liderazgo que tienda al perfeccionamiento del mismo". "La supervisión escolar es la expresión máxima del liderazgo educacional en acción. La Supervisión Escolar apunta al mejoramiento del proceso de enseñanza aprendizaje, para lo cual tiene que tomar encuentra toda la estructura teórica, material y humana de la escuela".

Anne Hick, citado por Alain (2000) "La supervisión escolar debe entenderse como orientación profesional y asistencia dadas a personas competentes en materia de educación, cuando y donde sean necesarias, tendientes al perfeccionamiento de la situación total de enseñanza - aprendizaje"

San H. Moorer (1969) "En la educación moderna se utiliza el término supervisión para describir las actividades que se ocupan principal y directamente del estudio y mejoramiento de las condiciones que rodean el aprendizaje y la formación de alumnos y maestros"

Borton y Brueckner (1969) "La supervisión moderna es un servicio técnico experto destinado fundamentalmente a estudiar y mejorar cooperativamente todos los factores que influyen en el crecimiento y desarrollo del niño".

Neagley y Evans (1969) "La supervisión moderna es una acción positiva dinámica y democrática que tiene por objeto mejorar la educación en el aula mediante el perfeccionamiento continuo de todas las personas involucradas: el niño, el docente, el supervisor, el administrador, el progenitor o cualquier otra persona legal".

Florencio Chacón (2000) "La supervisión docente es una actividad permanente que tiene por objeto conocer como realizan los docentes la función educativa, 
para ayudarlos y orientarlos en el trabajo; estimular a los educadores cuando lo hacen bien y muestran experiencias que puedan aprender los demás; entrenarlos para que interpreten y ejecuten las instrucciones que reciben; prepararlos para que acepten las decisiones de sus superiores $\mathrm{y}$, finalmente actualizarlos para que puedan adaptar su comportamiento a los cambios del mundo moderno y la dinámica como éstos ocurren".

\section{Objetivo General}

Determinar la relación existente entre la supervisión educativa y el desempeño docente en las instituciones educativas de la Red Nº8 - Puente Piedra. Lima 2014.

\section{Objetivos Específicos}

1. Determinar la relación entre la supervisión educativa y la preparación para la enseñanza en las Instituciones Educativas de la Red № 08 - Puente Piedra. Lima 2014.

2. Determinar la relación entre la supervisión educativa y la creación de un ambiente propicio en las Instituciones Educativas de la Red Nº8 - Puente Piedra. Lima 2014.

3. Determinar la relación entre la supervisión educativa y la enseñanza para el aprendizaje en las Instituciones Educativas de la Red Nº8 - Puente Piedra. Lima 2014.

4. Determinar la relación entre la supervisión educativa y el profesionalismo docente en las Instituciones Educativas de la Red № 08 - Puente Piedra. Lima 2014

Justificación: Se considera que los aportes teóricos de este estudio pueden constituir insumos relevantes respecto a la supervisión educativa y el desempeño docente, por lo tanto, coadyuvará en la generación de políticas de recurso humano, según las necesidades y exigencias educativas actuales. El trabajo de investigación aportará información útil para el mejoramiento de las funciones de los directores y de todos los docentes de las instituciones 
educativas en estudio, analizando el pasado, recomendando estrategias correctivas para ser aplicadas en la toma de decisiones.

\section{Resultados y Discusión:}

\section{Gráfico $N^{\circ} 1$. Supervisión Educativa}

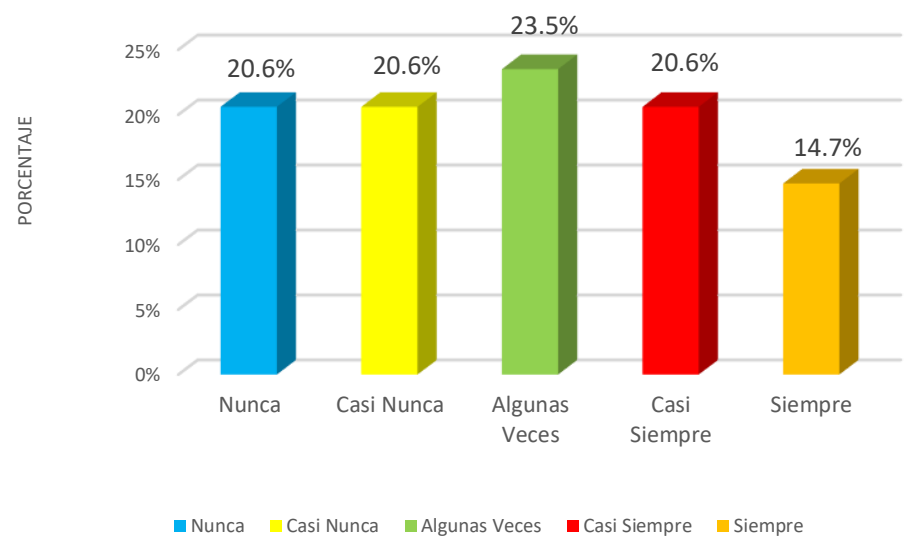

Fuente: Propia

Como se puede observar que la respuesta más frecuente es ALGUNAS VECES con un $23,53 \%$ y la menos frecuente es $14,71 \%$ que la respuesta es SIEMPRE, observamos también que en el porcentaje acumulado es el 64,7\% que representa a las respuestas NUNCA, CASI NUNCA y A VECES. También observamos que uno de cada cinco eligió CASI SIEMPRE.

\section{Gráfico N². Preparación para la Enseñanza}

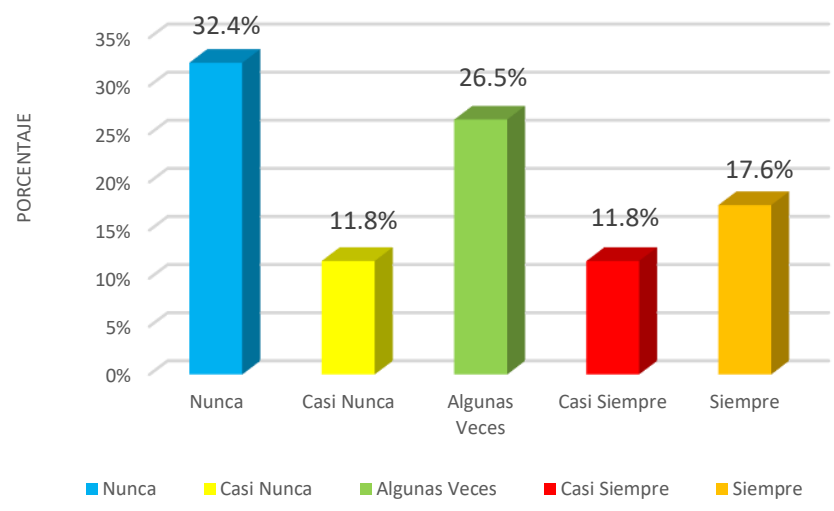

Fuente: Propia 
Como se puede observar que la respuesta más frecuente es NUNCA con un $32,35 \%$ y la menos frecuente es las respuestas CASI NUNCA y CASI SIEMPRE con un $11,76 \%$, observamos también que en el porcentaje acumulado es el $70,6 \%$ que representa a las respuestas NUNCA, CASI NUNCA y A VECES.

\section{Gráfico № 3. Creación de un ambiente de clase propicio}

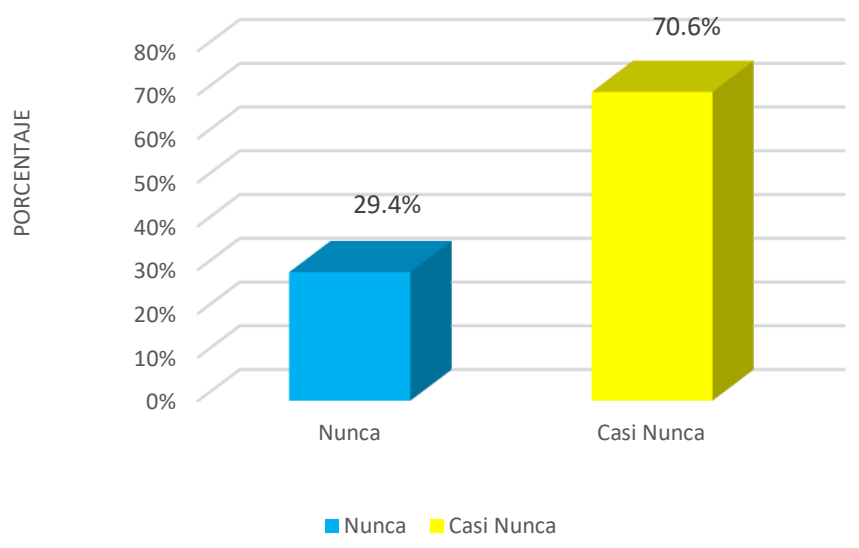

Fuente: Propia

Como se observa que la respuesta más frecuente es CASI NUNCA con un $70,59 \%$ y la menos frecuente es NUNCA con un $29,41 \%$

\section{Gráfico $\mathbf{N}^{\circ} 4$. Enseñanza para el aprendizaje}

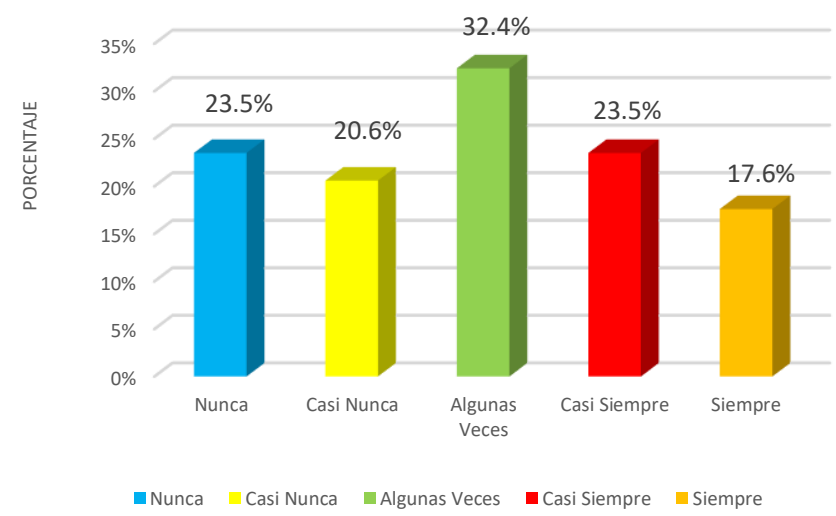

Fuente: Propia 
Como se puede observar que la respuesta más frecuente es ALGUNAS VECES con un $32,35 \%$ y la menos frecuente es la respuesta CASI NUNCA con un $20,59 \%$ cuyas respuestas son CASI NUNCA, observamos también que en el porcentaje acumulado es el $76,5 \%$ que representa a las respuestas NUNCA, CASI NUNCA y A VECES. Observamos que uno de cada 3 eligió ALGUNAS VECES.

\section{Gráfico $N^{\circ}$ 5. Profesionalismo Docente}

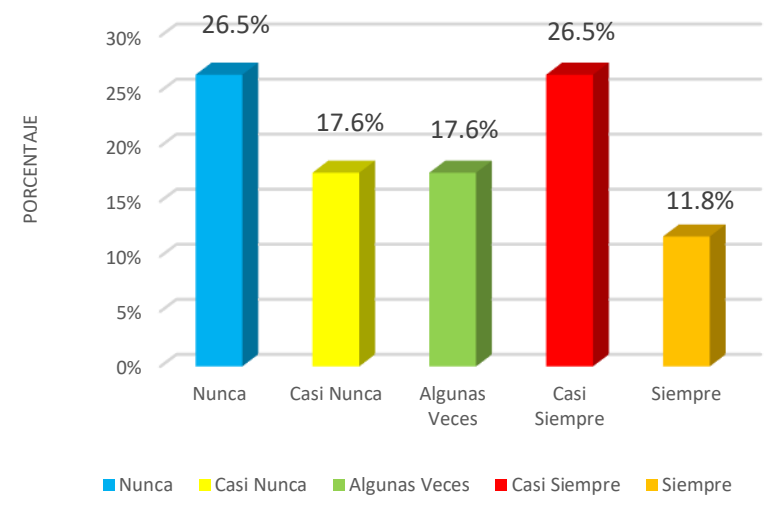

Fuente: Propia

Como se puede observar que la respuesta más frecuente es NUNCA y CASI SIEMPRE con un $26,47 \%$ y la menos frecuente es la respuesta SIEMPRE con un $11,76 \%$, observamos también que en el porcentaje acumulado es el $61,8 \%$ que representa a las respuestas NUNCA, CASI NUNCA y A VECES.

\section{Conclusiones:}

1. Se ha demostrado que existe relación significativa entre la gestión educativa y el buen desempeño docente en las instituciones educativas de la Red № 08 - Puente Piedra, esta decisión se sustenta en el valor de $\mathrm{p}=000$ y el rho calculado de 0,903 y de conformidad de lo establecido en la regla de decisión, se acepta la hipótesis de investigación, es decir, existe relación significativa entre la gestión educativa y el buen 
desempeño docente en las instituciones educativas de la Red Nº8 Puente Piedra 2014.

2. Encontramos que existe relación significativa entre la Gestión Educativa y el ambiente de clase en las Instituciones Educativas de la Red $N^{\circ} 08$ Puente Piedra, esta decisión se sustenta en el valor de $p=0,615$ y el rho calculado de 0,000 y de conformidad de lo establecido en la regla de decisión, se acepta la hipótesis nula, es decir, existe relación significativa entre la Gestión Educativa y el ambiente de clase en las Instituciones Educativas de la Red Nº8 Puente Piedra 2014.

3. Hemos demostrado que existe relación significativa entre la gestión educativa y la instrucción docente en las Instituciones Educativas de la Red N 08 Puente Piedra, esta decisión se sustenta en el valor de $p=000$ y el rho calculado de 0,766 y de conformidad de lo establecido en la regla de decisión, se acepta la hipótesis de investigación, es decir, Existe relación significativa entre la gestión educativa y la instrucción docente en las Instituciones Educativas de la Red Nº8 Puente Piedra 2014.

4. Los resultados demuestran que Existe relación significativa entre la Gestión Educativa y la responsabilidades docentes en las Instituciones Educativas de la Red № 08 Puente Piedra, esta decisión se sustenta en el valor de $\mathrm{p}=000$ y el rho calculado de 0,517 y de conformidad de lo establecido en la regla de decisión, se acepta la hipótesis de investigación, es decir, Existe relación significativa entre la Gestión Educativa y la responsabilidades docentes en las Instituciones Educativas de la Red Nº8 Puente Piedra 2014.

\section{Referencias Bibliográficas}

Fernández, F. (1992). Relación entre el Estilo Gerencial del Personal Directivo de las Escuelas Básicas del Altagracia de Orituco de la $3^{\text {a }}$ Etapa, y el 
Clima Organizacional de Dichas Instituciones. Trabajo de Maestría. Universidad Bicentenaria de Aragua. Venezuela

Ascanio E. (1995). El Liderazgo del Supervisor y la Motivación hacia el Mejoramiento Profesional en los Docentes que Laboran en la Tercera Etapa de Educación Básica. Trabajo de Grado para optar al título de Magister. Universidad "Rafael Urdaneta”. INSTIA. Caracas. Venezuela Lemus, L.A. (1975) Administracion, Direccion Y Supervision De Escuelas. Alain, A. (2000) Supervisión Educativa. Lima. Perú ABEDUL San H. Moorer (1969). La supervisión moderna. Edit. Omega. México D.F. Borton y Brueckner (1969) La supervisión moderna. Edit. Omega. México D.F. Neagley y Evans (1969) La supervisión moderna. Edit. Omega. México D.F. Chacón, F. (2002) Sistema de Control de Calidad. San José, Costa Rica. Universidad Estatal a Distancia. 